\title{
LITTLEWOOD'S PROBLEM FOR SETS WITH MULTIDIMENSIONAL STRUCTURE
}

\author{
BRANDON HANSON
}

\begin{abstract}
We give $L^{1}$-norm estimates for exponential sums of a finite sets $A$ consisting of integers or lattice points. Under the assumption that $A$ possesses sufficient multidimensional structure, our estimates are stronger than those of McGehee-Pigno-Smith and Konyagin. These theorems improve upon past work of Petridis.
\end{abstract}

\section{INTRODUCTION}

Let $A$ be a finite set of integers. The relationship between the additive structure of $A$ and the exponential sum 1

$$
F(t)=\sum_{a \in A} e(a t)
$$

is well-documented (see [TV], Chapter 4). In particular, we have

$$
\int_{0}^{1}|F(t)|^{2 k} d t=\left|\left\{\left(a_{1}, \ldots, a_{2 k}\right) \in A^{2 k}: a_{1}+\cdots+a_{k}=a_{k+1}+\cdots+a_{2 k}\right\}\right|,
$$

so that the larger the $2 k^{\prime}$ th moment of $F$, the more additive structure $A$ possesses. For $1 \leq p<2$, we expect the $L^{p}$-norms of $F$ to be smaller for additively structured sets. This led Littlewood to conjecture (see, for instance [HL) that

$$
\inf _{\substack{A \subseteq \mathbb{Z} \\|A|=N}} \int_{0}^{1}\left|\sum_{a \in A} e(a t)\right| d t=\int_{0}^{1}\left|\sum_{n=1}^{N} e(n t)\right| d t .
$$

It was a great feat when the estimate

$$
\inf _{\substack{A \subseteq \mathbb{Z} \\|A|=N}} \int_{0}^{1}\left|\sum_{a \in A} e(a t)\right| d t \geq C \int_{0}^{1}\left|\sum_{n=1}^{N} e(n t)\right| d t
$$

was established by McGehee, Pigno and Smith in [MPS] and independently by Konyagin in [Ko] for some absolute constant $C>0$. Here we will record the theorem of McGehee, Pigno and Smith as it will be used repeatedly in this article.

Theorem 1.1. Let $a_{1}<\ldots<a_{n}$ be a sequence of integers and let $u_{1}, \ldots, u_{n}$ be complex numbers. Then

$$
\int_{0}^{1}\left|\sum_{j=1}^{n} u_{j} e\left(a_{j} t\right)\right| d t \geq C_{M P S} \sum_{j=1}^{n} \frac{\left|u_{j}\right|}{j}
$$

\footnotetext{
${ }^{1}$ Throughout, we will use the notation $e(z)=e^{2 \pi i z}$.
} 
where $C_{M P S}>0$ is an absolute constant.

The estimate (21) leaves open a few questions. First, it remains to establish the sharp constant, i.e. to prove (11). Second, given a positive constant $C$, one would like to characterize the sets $A$ for which

$$
\int_{0}^{1}\left|\sum_{a \in A} e(a t)\right| d t \leq C \int_{0}^{1}\left|\sum_{n=1}^{N} e(n t)\right| d t,
$$

a question sometimes referred to as the Inverse Littlewood Problem, see [G]. This article concerns the latter problem and we interpret it as follows: if $A$ possesses some structure which is decidedly unlike an arithmetic progression, can the estimate (2) be improved? Specifically, we will explore how the notion of dimension can be leveraged. Such questions have already been investigated by Petridis $[\mathrm{P}]$, and where appropriate we will compare results.

The first notion of dimension we will explore is quite literal - we consider $A$ a subset of the lattice $\mathbb{Z}^{r}$. Since $\mathbb{Z}^{r}$ contains one-dimensional sets, one must take steps to ensure $A$ is truly multidimensional, which we now do.

For $i=1, \ldots, r$, let $\pi_{i}: \mathbb{Z}^{r} \rightarrow \mathbb{Z}$ denote the $i$ 'th coordinate projection and let

$$
A_{i}=\pi_{i}(A)
$$

denote the image of $A$ under this projection; for $a_{i} \in \pi_{i}(A)$ let

$$
A_{i}^{*}\left(a_{i}\right)=\pi_{i}^{-1}\left(a_{i}\right) \cap A
$$

denote the fibre of $A$ above $a_{i}$. Our first estimate extends Theorem 1.1 to higher dimensional sets.

Theorem 1.2. Suppose $A \subseteq \mathbb{Z}^{r}$ and $A_{1}$ is ordered as

$$
A_{1}=\left\{a_{1,1}<\ldots<a_{1, n}\right\} .
$$

Then we have the estimate

$$
\int_{[0,1]^{r}}\left|\sum_{\boldsymbol{a} \in A} e(\boldsymbol{a} \cdot \boldsymbol{t})\right| d \boldsymbol{t} \geq C_{M P S} \sum_{j=1}^{n} \frac{1}{j} \int_{[0,1]^{r-1}}\left|\sum_{a^{*} \in A_{1}^{*}\left(a_{1, j}\right)} e\left(\boldsymbol{a}^{*} \cdot \boldsymbol{t}\right)\right| d \boldsymbol{t} .
$$

We say $A \subseteq \mathbb{Z}$ is $n$-strongly 1 -dimensional if $|A| \geq n$. Inductively, if $\left(n_{1}, \ldots, n_{r}\right)$ is a $r$-tuple of natural numbers then we say a set $A \subseteq \mathbb{Z}^{r}$ is $\left(n_{1}, \ldots, n_{r}\right)$-strongly $r$-dimensional if $\left|A_{1}\right| \geq n_{1}$ and $\left|A_{1}^{*}\left(a_{1}\right)\right|$ is $\left(n_{2}, \ldots, n_{r}\right)$-strongly $(r-1)$-dimensional for each $a_{1} \in A_{1}$.

Theorem 1.3. Suppose $A \subseteq \mathbb{Z}^{r}$ is a $\left(n_{1}, \ldots, n_{r}\right)$-strongly $r$-dimensional subset of $\mathbb{Z}^{r}$. Then

$$
\int_{[0,1]^{r}}\left|\sum_{\boldsymbol{a} \in A} e(\boldsymbol{a} \cdot \boldsymbol{t})\right| d \boldsymbol{t} \geq C_{M P S}^{r} \log \left(n_{1}\right) \cdots \log \left(n_{r}\right) .
$$


Here we have strived to make the dependence on the implicit constant from Theorem 1.1 explicit. This estimate is an improvement on Theorem $1.2 \mathrm{in}[\mathrm{P}]$ and is best-possible up to the constant $C_{M P S}^{r}$.

We now move to the case of subsets $A$ of $\mathbb{Z}$, which are one dimensional but have a structure if higher dimensional sets. As motivation, recall that a generalized arithmetic progression of rank 2 is a set of the form

$$
G=\{a m+b n: 1 \leq m \leq M, 1 \leq n \leq N\}
$$

These sets arise as projections of boxes in $\mathbb{Z}^{2}$, hence we think of them as possessing multidimensional structure. To guarantee that the elements $a m+b n$ are distinct, it is sufficient to impose the condition $a M<b$. It is this sort of condition, which can be viewed as a multiscale condition, that motivates the last theorem of this article. To state it, we begin with appropriate notions of a multidimensional subset of $\mathbb{Z}$. We say $A \subseteq \mathbb{Z}$ is $n$ strongly 1-dimensional if $|A| \geq n$. For $\delta_{1}, \ldots, \delta_{r-1}>0$, we define inductively that a finite set $A \subseteq \mathbb{Z}$ is $\left(\delta_{1}, \ldots, \delta_{r-1} ; n_{1}, \ldots, n_{r}\right)$-strongly $r$-dimensional if there are numbers $d_{1}$ and $d_{2}$ with $d_{2}>\left(2+\delta_{1}\right) d_{1}$ and such that

$$
A=\bigcup_{k \in I} A_{k}+k d_{2}
$$

for some set $I$ of consisting of at least $n_{1}$ integers and subsets $A_{k} \subseteq\left\{-d_{1}, \ldots, d_{1}\right\}$ which are each $\left(\delta_{2}, \ldots, \delta_{r-1} ; n_{2}, \ldots, n_{r}\right)$-strongly $(r-1)$-dimensional.

To get a sense of this definition, it is best to think of strongly 2-dimensional sets; higher dimensional sets are handled by an iterative argument. The easiest example of a $(\delta, n)$ strongly 2-dimensional set is a union of intervals $A_{k}$ of length $n$, separated by gaps of length $\delta n$. One needs that $n$ is somewhat large - if $n$ were 1 , then this set would be an arithmetic progression; one also requires the gaps between intervals - without them, our set would be an ordinary interval. However our definition allows, for instance, to pass to very sparse subsets of such a set. In general, each set $A_{k}$ can be compared to the fibres $A_{1}^{*}\left(a_{k}\right)$ in the $\mathbb{Z}^{2}$ setting, and then we only require that these fibres are sufficiently large (but independently of $d_{1}$ ). The condition that $d_{2}>(2+\delta) d_{1}$ imposes a gap of size at least $\delta d_{1}$ between any two $A_{k}$. This gap is a substitute for the fact that the fibres of a projection $\pi: \mathbb{Z}^{2} \rightarrow \mathbb{Z}$ are independent.

Theorem 1.4. Let $\delta, \ldots, \delta_{r-1}>0$ and $n_{1}, \ldots, n_{r}$ be positive integers satisfying

$$
n_{i} \geq \pi^{3} 2^{21} C_{M P S}^{3} \prod_{j=i}^{r}\left(\log \left(n_{j}\right)\right)^{3}
$$

for each $i$. Suppose $A$ is a $\left(\delta_{1} \ldots, \delta_{r-1} ; n_{1}, \ldots, n_{r}\right)$-strongly $r$-dimensional subset of $\mathbb{Z}$. Then

$$
\int_{0}^{1}\left|\sum_{a \in A} e(a t)\right| d t \geq C_{\delta_{1} \ldots, \delta_{r-1}} \log \left(n_{1}\right) \cdots \log \left(n_{r}\right),
$$


where

$$
C_{\delta_{1} \ldots, \delta_{r-1}}=C_{M P S}^{r}\left(2^{9} \pi\right)^{-r} \prod_{j=1}^{r-1}\left(2+\log \left(1+2 / \delta_{j}\right)\right)^{-1} .
$$

This theorem is also best-possible up to the constant, as the lower bound is realized by an appropriately chosen $r$-dimensional arithmetic progression, see Theorem 3.3 in [S]. Estimates for multidimensional subsets of $\mathbb{Z}$ were established in Theorem 1.3 of $[\mathrm{P}]$ as well. There, the bounds are likely not as sharp as in Theorem 1.4, but the hypotheses are somewhat different, relying on the notion of a Freiman isomorphism. It might also be noted that Theorem 1.4 holds for two-dimensional sets, which was not established in $[\mathrm{P}]$.

\section{ACKNOWLEDGEMENTS}

I am grateful for the help of Giorgis Petridis and Ákos Magyar. They generously contributed ideas which improved the arguments in this article.

\section{Strongly MULTidimensional SETS In $\mathbb{Z}^{r}$}

The basic argument relies on the solution to Littlewood's problem by McGehee-PignoSmith, and in particular their generalized Hardy inequality.

Proof of Theorem 1.2. Let $\left(t_{1}, t_{2}, \ldots, t_{r}\right) \in[0,1]^{r}$ and write

$$
\sum_{\boldsymbol{a} \in A} e(\boldsymbol{a} \cdot \boldsymbol{t})=\sum_{j=1}^{n} e\left(a_{1, j} t_{1}\right)\left(\sum_{\boldsymbol{a}^{*} \in A_{1}^{*}\left(a_{1}\right)} e\left(\boldsymbol{a}^{*} \cdot\left(t_{2}, \ldots, t_{r}\right)\right)\right) .
$$

We interpret this as a trigonometric polynomial in the variable $t_{1}$ with complex coefficients. By Theorem 1.1 we have

$$
\int_{0}^{1}\left|\sum_{\boldsymbol{a} \in A} e(\boldsymbol{a} \cdot \boldsymbol{t})\right| d t_{1} \geq C_{M P S} \sum_{j=1}^{n} \frac{1}{j}\left|\sum_{\boldsymbol{a}^{*} \in A_{1}^{*}\left(a_{1}\right)} e\left(\boldsymbol{a}^{*} \cdot\left(t_{2}, \ldots, t_{r}\right)\right)\right| .
$$

Integrating over $t_{2}, \ldots, t_{r}$ completes the proof.

Iterated application of Theorem 1.2 leads to the proof of Theorem 1.3.

Proof of Theorem 1.3. We proceed by induction on $r$, and when $r=1$, this follows immediately from Theorem 1.1. By the preceding proposition, we have

$$
\int_{[0,1]^{d}}\left|\sum_{\boldsymbol{a} \in A} e(\boldsymbol{a} \cdot \boldsymbol{t})\right| d \boldsymbol{t} \geq C_{M P S} \sum_{j=1}^{n} \frac{1}{j} \int_{[0,1]^{r-1}}\left|\sum_{\boldsymbol{a}^{*} \in A_{1}^{*}\left(a_{1}\right)} e\left(\boldsymbol{a}^{*} \cdot \boldsymbol{t}\right)\right| d \boldsymbol{t},
$$

and each of the sets $A_{1}^{*}\left(a_{1}\right)$ is $\left(n_{2}, \ldots, n_{r}\right)$-strongly $(r-1)$-dimensional. By induction,

$$
\int_{[0,1]^{r-1}}\left|\sum_{\boldsymbol{a}^{*} \in A_{1}^{*}\left(a_{1}\right)} e\left(\boldsymbol{a}^{*} \cdot \boldsymbol{t}\right)\right|_{4} d \boldsymbol{t} \geq C_{M P S}^{r-1} \log \left(n_{2}\right) \cdots \log \left(n_{r}\right)
$$


and since $n \geq r_{1}$ the theorem is proved.

\section{Lemmata}

Throughout, we will say $f$ is a trigonometric polynomial of degree $d$ if

$$
f(t)=\sum_{|n| \leq d} a_{n} e(n t)
$$

Notice $L^{1}$-norms are preserved if we translate the support of $\widehat{f}$ by $d$. We need the following.

Lemma 3.1 (Bernstein's inequality). Let $f:[0,1] \rightarrow \mathbb{C}$ be a trigonometic polynomial of degree $d$. Then

$$
\left\|f^{\prime}\right\|_{L^{1}([0,1])} \leq 2 \pi d\|f\|_{L^{1}([0,1])} .
$$

Proof. See [Ka, Chapter 1, Excercise 7.16].

Lemma 3.2. Let $n$ be a positive integer. Then for any trigonometric polynomial $f$ of degree $d$ we have

$$
\left|\|f\|_{L^{1}([0,1])}-\frac{1}{N} \sum_{j=1}^{N}\right| f(j / N)|| \leq \frac{4 \pi d}{N}\|f\|_{L^{1}([0,1])} .
$$

Proof. We begin with the estimate,

$$
\left|\int_{0}^{1}\right| f(t)\left|d t-\frac{1}{N} \sum_{j=1}^{N}\right| f(j / N)|| \leq \frac{1}{N} \operatorname{Var}(|f|)
$$

where

$$
\operatorname{Var}(g)=\sup _{0=x_{0}<x_{1}<\ldots<x_{M}=1} \sum_{j=1}^{M}\left|g\left(x_{j}\right)-g\left(x_{j-1}\right)\right|
$$

is the total variation of $g$. This is a simple case of, for instance, Koksma's inequality, see [KN. Since

$$
\operatorname{Var}(|f|) \leq \operatorname{Var}(\Re(f))+\operatorname{Var}(\Im(f))=\int_{0}^{1}\left|\Re(f)^{\prime}(t)\right| d t+\int_{0}^{1}\left|\Im(f)^{\prime}(t)\right| d t \leq 2 \int_{0}^{1}\left|f^{\prime}(t)\right| d t
$$

the result now follows from Bernstein's inequality.

One of the key ideas that goes into the proof of Theorem 1.4 is to amplify the gaps between the different pieces of $A$. To do so, we need a function that can isolate the various subsets $A_{k}$.

Lemma 3.3. Let $M$ and $N$ be integers with $2 \leq M<N$ and let $R \geq 2 N+4 M+1$. Then there is a function $K_{M, N}$ with the following properties:

(1) $K_{M, N}(k)=1$ for $|k| \leq N$,

(2) $K_{M, N}(k)=0$ for $|k| \geq N+2 M$, and 


$$
\frac{1}{R} \sum_{j=1}^{R}\left|\widehat{K_{M, N}}(j / R)\right| \leq 32 \pi(2+\log (1+N / M)) .
$$

Proof. Recall that the Dirichlet kernel of order $N$ is

$$
D_{N}(t)=\sum_{|n| \leq N} e(n t)=\frac{\sin (\pi(2 N+1) t)}{\sin (\pi t)},
$$

and the Fejer kernel or order $N$ is

$$
F_{N}(t)=\sum_{|n| \leq N}\left(1-\frac{|n|}{N+1}\right) e(n t)=\frac{1}{N+1} \frac{(\sin (\pi(N+1) t))^{2}}{(\sin (\pi t))^{2}} .
$$

We have

$$
\left|D_{N}(t)\right| \leq 2 N+1,0 \leq F_{N}(t) \leq N+1, \int_{0}^{1} F_{N}(t) d t=1 .
$$

Now let $M$ and $N$ be integers with $M<N$ and define

$$
K_{M, N}(k)=\frac{1}{M} \sum_{\substack{|n| \leq M-1 \\|n-k| \leq N+M}}\left(1-\frac{|n|}{M}\right) .
$$

Then

$$
\widehat{K_{M, N}}(t)=\frac{1}{M} D_{N+M}(t) F_{M-1}(t) .
$$

First, observe that if $|k| \leq N$ then

$$
K_{M, N}(k)=\frac{1}{M} \sum_{|n| \leq M-1}\left(1-\frac{|n|}{M}\right)=1 .
$$

Meanwhile if $|k| \geq N+2 M$ then $K_{M, N}(k)=0$ since the defining sum is empty. Thus we have (1) and (2).

For (3), we have

$$
\int_{0}^{1}\left|\widehat{K_{M, N}}(t)\right| d t=2 I_{1}+2 I_{2}+2 I_{3}
$$

where

$$
\begin{gathered}
I_{1}=\frac{1}{M} \int_{0}^{\frac{1}{N+M}}\left|D_{M+N}(t)\right| F_{M-1}(t) d t \leq 3, \\
I_{2}=\frac{1}{M} \int_{\frac{1}{N+M}}^{\frac{1}{M}}\left|D_{M+N}(t)\right| F_{M-1}(t) d t,
\end{gathered}
$$

and

$$
I_{3}=\frac{1}{M} \int_{\frac{1}{M}}^{\frac{1}{2}}\left|D_{M+N}(t)\right| F_{M-1}(t) d t .
$$


Using $2 t \leq|\sin (\pi t)| \leq \pi t$ for $|t| \leq \frac{1}{2}$, we have

$$
\begin{aligned}
I_{2} & \leq \frac{1}{M^{2}} \int_{\frac{1}{N+M}}^{\frac{1}{M}} \frac{|\sin (\pi M t)|^{2}}{|\sin (\pi t)|^{3}} d t \\
& \leq \frac{\pi^{2}}{8} \int_{\frac{1}{N+M}}^{\frac{1}{M}} \frac{d t}{t} \\
& \leq 2 \log (1+N / M) .
\end{aligned}
$$

Finally,

$$
I_{3} \leq \frac{1}{M^{2}} \int_{\frac{1}{M}}^{\frac{1}{2}} \frac{1}{t^{3}} d t \leq 1
$$

By Lemma 3.2,

$$
\frac{1}{R} \sum_{j=1}^{R}\left|\widehat{K_{M, N}}(j / R)\right| \leq 4 \pi\left\|\widehat{K_{M, N}}\right\|_{L^{1}([0,1])} \leq 8 \pi(4+2 \log (1+N / M)) .
$$

Lemma 3.4. Let $R$ a positive integer and $K: \mathbb{Z} \rightarrow \mathbb{C}$ be a periodic function with period $R$.

Then

$$
\int_{0}^{1}\left|\sum_{m} a_{m} K(m) e(m t)\right| d t \leq \frac{1}{R} \sum_{j=1}^{R}|\widehat{K}(j / R)| \int_{0}^{1}\left|\sum_{m} a_{m} e(m t)\right| d t .
$$

Proof. By orthogonality of characters modulo $R$

$$
\sum_{m} a_{m} K(m) e(m t)=\frac{1}{R} \sum_{j=1}^{R} \widehat{K}(j / R) \sum_{m} a_{m} e(m(t+j / R)) .
$$

So by the triangle inequality,

$$
\int_{0}^{1}\left|\sum_{m} a_{m} K(m) e(m t)\right| d t \leq \frac{1}{R} \sum_{j=1}^{R}|\widehat{K}(j / R)| \int_{0}^{1}\left|\sum_{m} a_{m} e(m(t+y / R))\right| d t .
$$

Given a set $I \subseteq \mathbb{Z}$, a positive integer $q$, and an arbitrary integer $s$, we define

$$
I(q ; s)=\{k \in I: k=s(\bmod q)\} .
$$

The following lemma is used to amplify the space between the sets $A_{k}$.

Lemma 3.5. Let $d_{1}, d_{2}$ and $q$ be positive integers with $(2+2 \delta) d_{1}+4 \leq d_{2}$ for some $\delta>0$ and $q \geq 4$. Suppose $I$ is a finite set of integers, and let

$$
F(t)=\sum_{k \in I} f_{k}(t) e\left(d_{2} k t\right)
$$


where each $f_{k}$ is a trigonometric polynomial of degree at most $d_{1}$. Then for any integer $s$, we have

$$
\int_{0}^{1}\left|\sum_{k \in I(q ; s)} f_{k}(t) e\left(d_{2} k t\right)\right| d t \leq 32 \pi(2+\log (1+2 / \delta))\|F\|_{L^{1}([0,1])} .
$$

Proof. If necessary we may replace $I$ with $I-s$ while preserving the $L^{1}$-norm, and so there is no loss of generality in assuming $s=0$. By definition, we can write

$$
F(t)=\sum_{m} a_{m} e(m t)
$$

where the coefficients $a_{m}$ are supported on numbers of the form

$$
m=d_{2} k+l,|l| \leq d_{1} .
$$

Let $M=\left\lceil\delta d_{1} / 2\right\rceil$ and $N=d_{1}$, and let $K_{M, N}$ be the function from Lemma 3.3. The support of $K_{M, N}$ is contained in the interval

$$
[-N-2 M, N+2 M] \subseteq\left[-d_{2} / 2, d_{2} / 2\right]
$$

and $K_{M, N}$ is identically 1 on $\left\{-d_{1}, \ldots, d_{1}\right\}$. Extend $K_{M, N}$ periodically with period $q d_{2}$. Then by Lemma 3.4,

$$
\int_{0}^{1}\left|\sum_{m} a_{m} K_{M, N}(m) e(m t)\right| d t \leq 32 \pi(2+\log (1+N / M))\|F\|_{L^{1}([0,1])} .
$$

Now $a_{m} K_{M, N}(m)$ is only non-zero if $m=j q d_{2}+l^{\prime}$ with $-d_{2} / 2 \leq l^{\prime} \leq d_{2} / 2$. By (3), we have

$$
l-l^{\prime}=d_{2}(j q-k)
$$

and since $\left|l-l^{\prime}\right| \leq d_{1}+d_{2} / 2<d_{2}$, this can only happen if $k=j q$ and $l=l^{\prime}$. So we are left with coefficients supported on integers of the form $j q d_{2}+l$ with $-d_{1} \leq l \leq d_{1}$. However, $K_{M, N}$ is identically 1 on numbers of the form $j q d_{2}+l$ with $-d_{1} \leq l \leq d_{1}$. In summary,

$$
\sum_{m} a_{m} K_{M, N}(m) e(m t)=\sum_{\substack{k \in I \\ k=0}(\bmod q)} e\left(k d_{2} t\right) \sum_{|l| \leq d_{1}} a_{k d_{2}+l} e(l t)=\sum_{k \in I(q ; 0)} f_{k}(t) e\left(k d_{2} t\right) .
$$

Finally, in order to apply Lemma 3.5 effectively, we need a good modulus $q$. Such a modulus is guaranteed by the following lemma.

Lemma 3.6. Let $I$ be a set integers with $|I| \geq 8$. Then there are positive integers $q$ and $s$ such that

$$
|I|^{1 / 3} / 8 \leq \underset{8}{|I(q ; s)|} \leq q^{1 / 2}
$$


Proof. For each $j \geq 1$, choose any $s_{j}$ so that $\left|I\left(4^{j} ; s_{j}\right)\right|$ is maximal. Then $\left|I\left(4^{j} ; s_{j}\right)\right| \geq 4^{-j}|I|$ by the pigeonhole principle. We have

$$
\left|I\left(4 ; s_{1}\right)\right| \geq|I| / 4 \geq 2
$$

while for sufficiently large $j$ we have $\left|I\left(4^{j} ; s_{j}\right)\right|=1 \leq 2^{j}$. It follows that there is a minimal $j_{0}$ so that $\left|I\left(4^{j_{0}} ; s_{j_{0}}\right)\right| \leq 2^{j_{0}}$. We let $q=4^{j_{0}}$, and $s=s_{j_{0}}$. Then

$$
\frac{|I|}{q} \leq|I(q, s)| \leq q^{1 / 2}
$$

so that in particular $|I|^{1 / 3} \leq q^{1 / 2}$. By minimality of $j_{0}$

$$
q^{1 / 2} / 2=2^{j_{0}-1} \leq\left|I\left(q / 4 ; s_{j_{0}-1}\right)\right| \leq 4 I(q ; s),
$$

so that $|I(q ; s)| \geq|I|^{1 / 3} / 8$.

\section{Multidimensional Subsets of $\mathbb{Z}$}

The following proposition can be viewed as a sort of analog to Theorem 1.2 .

Proposition 4.1. Let $d_{1}, d_{2}$ positive integers with $(2+\delta) d_{1}<d_{2}$. Suppose $I$ is a finite set of integers, and let

$$
F(t)=\sum_{k \in I} f_{k}(t) e\left(d_{2} k t\right)
$$

where

$$
f_{k}(t)=\sum_{|n| \leq d_{1}} a_{n, k} e(n t) .
$$

Let $q$ and $s$ an integers with $q>4 \pi$ and suppose

$$
I(q ; s)=\left\{k_{1}<\ldots<k_{J}\right\} .
$$

Then we have have

$$
\|F\|_{L^{1}([0,1])} \geq \frac{1}{32 \pi(2+\log (1+2 / \delta))} \sum_{j=1}^{J}\left\|f_{k_{j}}\right\|_{L^{1}([0,1])}\left(\frac{C_{M P S}}{2 j}-\frac{2 \pi d_{1}}{q d_{2}}\right) .
$$

Proof. By the Lemma 3.5, we have

$$
\|F\|_{L^{1}([0,1])} \geq \frac{1}{32 \pi(2+\log (1+2 / \delta))} \int_{0}^{1}\left|\sum_{j=1}^{J} f_{k_{j}}(t) e\left(k_{j} d_{2} t\right)\right| d t .
$$

Next we write each $k_{j} \in I(q ; s)$ as $k_{j}=b_{k_{j}} q+s$, so the above integral becomes

$$
\begin{aligned}
\int_{0}^{1}\left|\sum_{j=1}^{J} f_{k_{j}}(t) e\left(\left(b_{k_{j}} q+s\right) d_{2} t\right)\right| d t & =\int_{0}^{1}\left|\sum_{j=1}^{J} f_{k_{j}}(t) e\left(b_{k_{j}} q d_{2} t\right)\right| d t \\
& =\frac{1}{q d_{2}} \int_{0}^{q d_{2}}\left|\sum_{j=1}^{J} f_{k_{j}}\left(u / q d_{2}\right) e\left(b_{k_{j}} u\right)\right| d u .
\end{aligned}
$$


after the change of variables $u=q d_{2} t$. Next, by breaking the integral into intervals of unit length, we get

$$
\frac{1}{q d_{2}} \sum_{m=1}^{q d_{2}} \int_{m-1}^{m}\left|\sum_{j=1}^{J} f_{k_{j}}\left(u / q d_{2}\right) e\left(b_{k_{j}} u\right)\right| d u \geq T_{1}-T_{2}
$$

where

$$
T_{1}=\frac{1}{q d_{2}} \sum_{m=1}^{q d_{2}} \int_{m-1}^{m}\left|\sum_{j=1}^{J} f_{k_{j}}\left((m-1) / q d_{2}\right) e\left(b_{k_{j}} u\right)\right| d u
$$

and

$$
T_{2}=\frac{1}{q d_{2}} \sum_{m=1}^{q d_{2}} \int_{m-1}^{m}\left|\sum_{j=1}^{J}\left(f_{k_{j}}\left(u / q d_{2}\right)-f_{k_{j}}\left((m-1) / q d_{2}\right)\right) e\left(b_{k_{j}} u\right)\right| d u .
$$

By periodicity followed by Theorem 1.1, we have the estimate

$$
T_{1}=\frac{1}{q d_{2}} \sum_{m=1}^{q d_{2}} \int_{0}^{1}\left|\sum_{j=1}^{J} f_{k_{j}}\left((m-1) / q d_{2}\right) e\left(b_{k_{j}} u\right)\right| d u \geq \frac{C_{M P S}}{q d_{2}} \sum_{m=1}^{q d_{2}} \sum_{j=1}^{J} \frac{\left|f_{k_{j}}\left((m-1) / q d_{2}\right)\right|}{j} .
$$

An application of Lemma 3.2 , bearing in mind $q d_{2}>8 \pi d_{1}$, yields

$$
\frac{1}{q d_{2}} \sum_{m=1}^{q d_{2}}\left|f_{k_{j}}\left((m-1) / q d_{2}\right)\right| \geq \frac{1}{2}\left\|f_{k_{j}}\right\|_{L^{1}([0,1])}
$$

To complete the proof of the proposition it suffices to estimate $T_{2}$ appropriately. For $u$ in the interval $m-1 \leq u \leq m$ we have

$$
\begin{aligned}
\left|\sum_{j=1}^{J}\left(f_{k_{j}}\left(u / q d_{2}\right)-f_{k_{j}}\left((m-1) / q d_{2}\right)\right) e\left(b_{k_{j}} u\right)\right| & =\left|\sum_{j=1}^{J} \int_{\frac{m-1}{q d_{2}}}^{\frac{u}{q d_{2}}} f_{k_{j}}^{\prime}(v) e\left(b_{k_{j}} u\right) d v\right| \\
& \leq \int_{\frac{m-1}{q d_{2}}}^{\frac{m}{q d_{2}}}\left|\sum_{j=1}^{J} f_{k_{j}}^{\prime}(v) e\left(b_{k_{j}} u\right)\right| d v
\end{aligned}
$$

by the triangle inequality and positivity. Thus we get the upper bound

$$
\begin{aligned}
T_{2} & \leq \frac{1}{q d_{2}} \sum_{m=1}^{q d_{2}} \int_{\frac{m-1}{q d_{2}}}^{\frac{m}{q d_{2}}} \int_{m-1}^{m}\left|\sum_{j=1}^{J} e\left(b_{k_{j}} u\right) f_{k_{j}}^{\prime}(v)\right| d u d v \\
& =\frac{1}{q d_{2}} \int_{0}^{1} \int_{0}^{1}\left|\sum_{j=1}^{J} e\left(b_{k_{j}} u\right) f_{k_{j}}^{\prime}(v)\right| d u d v \\
& \leq \frac{1}{q d_{2}} \sum_{j=1}^{J} \int_{0}^{1}\left|f_{k_{j}}^{\prime}(v)\right| d v .
\end{aligned}
$$

By Lemma 3.1, we obtain

$$
T_{2} \leq \frac{2 \pi d_{1}}{q d_{2}} \sum_{j=1}^{J}\left\|f_{k_{j}}\right\|_{L^{1}([0,1])}
$$


Proof of Theorem 1.4. As in the proof of 1.3, we proceed by induction on $r$. When $r=1$, this follows immediately from Theorem 1.1.

For the inductive step, we begin by writing $A$ as

$$
A=\bigcup_{k \in I} A_{k}+k d_{2}
$$

for some $\left(n_{2}, \ldots, n_{r}\right)$-strongly regular sets $A_{k} \subseteq\left\{-d_{1}, \ldots, d_{1}\right\}$, where $d_{1}$ and $d_{2}$ with $16<$ $4 d_{1}<d_{2}$. If we let

$$
f_{k}(t)=\sum_{a \in A_{k}} e(a t)
$$

then

$$
\sum_{a \in A} e(a t)=\sum_{k \in I} f_{k}(t) e\left(d_{2} k t\right)
$$

is of the necessary form to apply Proposition 4.1. If it is the case that

$$
\left\|f_{k}\right\|_{L^{1}([0,1])} \geq C_{\delta_{2}, \ldots, \delta_{r-1}} \log \left(n_{1}\right) \cdots \log \left(n_{r}\right)
$$

for some $k$, then we can choose $q$ so large and $s$ in such a way that $I(q ; s)=\{k\}$. This gives that

$$
\|F\|_{L^{1}([0,1])} \geq \frac{C_{M P S}}{32 \pi\left(2+\log \left(1+2 / \delta_{1}\right)\right)}\left\|f_{k}\right\|_{L^{1}([0,1])}
$$

yielding the theorem immediately. Thus there is no loss of generality in assuming

$$
\left\|f_{k}\right\|_{L^{1}([0,1])} \leq C_{\delta_{2}, \ldots, \delta_{r-1}} \log \left(n_{1}\right) \cdots \log \left(n_{r}\right)
$$

for each $k \in I$.

Now we choose $q$ and $s$ as in Lemma 3.6 applied to $I$ to get

$$
I(q ; s)=\left\{k_{1}<\ldots<k_{J}\right\}
$$

satisfying

$$
\frac{n_{1}^{1 / 3}}{8} \leq J \leq q^{1 / 2}
$$

Then

$$
\int_{0}^{1}\left|\sum_{a \in A} e(a t)\right| d t \geq \frac{1}{2^{5} \pi(2+\log (1+2 / \delta))} \sum_{j=1}^{J}\left\|f_{k_{j}}\right\|_{L^{1}([0,1])}\left(\frac{C_{M P S}}{2 j}-\frac{2 \pi d_{1}}{q d_{2}}\right) .
$$

By induction,

$$
\sum_{j=1}^{J} \frac{\left\|f_{k_{j}}\right\|_{L^{1}([0,1])}}{j} \geq C_{\delta_{2}, \ldots, \delta_{r-1}} \log (J) \log \left(n_{2}\right) \cdots \log \left(n_{r}\right) \geq \frac{1}{4} C_{\delta_{2}, \ldots, \delta_{r-1}} \log \left(n_{1}\right) \cdots \log \left(n_{r}\right) .
$$

By (4), the error term in (5) is at most

$$
\frac{2 \pi J d_{1}}{q d_{2}} C_{\delta_{2}, \ldots, \delta_{r-1}} \log \left(n_{1}\right) \cdots \log \left(n_{r}\right) \leq \pi C_{\delta_{2}, \ldots, \delta_{r-1}} \frac{\log \left(n_{1}\right) \cdots \log \left(n_{r}\right)}{q^{1 / 2}} .
$$


As guaranteed by Lemma 3.6 and the hypotheses of the theorem,

$$
q^{1 / 2} \geq n_{1}^{1 / 3} / 8 \geq 16 \pi C_{M P S} \log \left(n_{1}\right) \cdots \log \left(n_{r}\right) .
$$

So, we have shown

$$
\begin{aligned}
\int_{0}^{1}\left|\sum_{a \in A} e(a t)\right| d t & \geq \frac{1}{2^{5} \pi(2+\log (1+2 / \delta))} \frac{C_{\delta_{2}, \ldots, \delta_{r-1}}}{2^{4}} \log \left(n_{1}\right) \cdots \log \left(n_{r}\right) \\
& =C_{\delta_{1}, \ldots, \delta_{r-1}} \log \left(n_{1}\right) \cdots \log \left(n_{r}\right) .
\end{aligned}
$$

\section{REFERENCES}

[G] B. J. Green, Approximate algebraic structure. Proceedings of the International Congress of MathematiciansSeoul 2014. Vol. 1, 341-367, Kyung Moon Sa, Seoul, 2014.

[Ka] Y. Katznelson, An introduction to harmonic analysis. Third edition. Cambridge Mathematical Library. Cambridge University Press, Cambridge, 2004.

[Ko] S. V. Konyagin, On the Littlewood problem. (Russian) Izv. Akad. Nauk SSSR Ser. Mat. 45 (1981), no. 2, 243-265, 463.

[KN] L. Kuipers L. and H. Niederreiter, Uniform distribution of sequences. Pure and Applied Mathematics. Wiley-Interscience, New York-London-Sydney, 1974.

[HL] G. H. Hardy and J. E. Littlewood, A new proof of a theorem on rearrangements. J. London Math. Soc. 23 (1948), 163-168.

[MPS] O. C. McGehee, L. Pigno, and B. Smith, Hardy's inequality and the $L^{1}$ norm of exponential sums. Ann. of Math. (2) 113 (1981), no. 3, 613-618.

[P] G. Petridis, The $L^{1}$-norm of exponential sums in $\mathbb{Z}^{d}$. Math. Proc. Cambridge Philos. Soc. 154 (2013), no. 3, 381-392.

[S] X. Shao, On character sums and exponential sums over generalized arithmetic progressions. Bull. Lond. Math. Soc. 45 (2013), no. 3, 541-550.

[TV] T. Tao and V. Vu, Additive combinatorics. Cambridge Studies in Advanced Mathematics, 105. Cambridge University Press, Cambridge, 2006.

University of Georgia, Athens, GA

E-mail address: brandon.w.hanson@gmail.com 